\title{
Explosion Diagnostics of Type Ia Supernovae from Early Infrared Spectra
}

\author{
J. Craig Wheeler, Peter Höflich, Robert P. Harkness \\ Department of Astronomy, University of Texas, Austin, TX78712, USA \\ and \\ Jason Spyromilio \\ European Southern Observatory, Karl-Schwarzschild-Strasse 2, D-85748, Garching bei München, Germany
}

\begin{abstract}
Models of infrared spectra of Type Ia supernovae around maximum light are presented. The underlying dynamic models are delayed detonation explosions in Chandrasekhar mass carbon/oxygen white dwarfs. In combination with the radiative transport codes employed here, these models provide plausible fits to the optical spectra of "normal" Type Ia supernova. Two independent radiative transport codes are used, one that assumes LTE and one that computes non-LTE excitations and ionization. The models are compared with infrared data available in the literature. The independent codes give a reasonable representation of the data and provide physical explanations for their origin independent of the detailed assumptions of the radiative transfer. The infrared gives an especially powerful diagnostic of the dynamic model because with strongly variable line blanketing opacity it probes different depths within the exploded white dwarf at the same epoch. The velocity of the transition zone between explosive oxygen and carbon burning can be directly determined. The velocity at which the burning to nickel stops can also be probed. These velocities are very sensitive to the explosion physics.
\end{abstract}

Subject headings: Supernovae: general, individual (SN1994D, SN1986G) — infrared — radiation transfer — line: identification, profiles 


\section{Introduction}

Infrared spectroscopy of supernovae at early times has been very sparse, mostly due to the limited number of facilities equipped with the appropriate instrumentation. As a result, the usefulness of such spectra as diagnostics of supernovae has not been investigated fully. The lack of models to appropriately explore these data has, in some cases, discouraged their publication. The lack of published data, in turn, has resulted in model spectra being artificially truncated at a wavelength of $1 \mu \mathrm{m}$. Here we will concentrate on interpretation of the IR spectral data of Type Ia supernovae (SN Ia).

Kirshner et al. (1973) and Branch et al. (1983) showed that after maximum in SN Ia the flux in J is depressed with respect to the continuum at longer and shorter wavelengths. Subsequent IR spectroscopy revealed a pronounced broad spectral minimum in the $\mathrm{J}$ band at $\sim 1.2 \mu \mathrm{m}$ (Frogel et al. 1987, Meikle et al. 1996) and other spectral features. Spectroscopic analysis of this early time data is limited. Meikle et al. (1996) concentrated on determining the origin of a feature at $1.05 \mu \mathrm{m}$ in the spectra of SN 1994D. They concluded that the feature might be due to either HeI $1.083 \mu \mathrm{m}$ or MgII $1.0926 \mu \mathrm{m}$, but found difficulties with both identifications. Lynch et al. (1990) were unable to determine a plausible explanation for the $1.2 \mu \mathrm{m}$ feature observed in a spectrum of SN 1989B. Graham (1986) attributed the $1.2 \mu \mathrm{m}$ spectroscopic feature to absorption by a multiplet of SiI. Höflich et al. (1993) showed that the line opacity has a deficit in the $1.2 \mu \mathrm{m}$ range but did not discuss the possible implications for the $1.2 \mu \mathrm{m}$ spectral feature. Spyromilio, Pinto \& Eastman (1994) argued that no multiplet or combination of lines is responsible for the lack of flux in the $1.2 \mu \mathrm{m}$ spectral region. Instead, it is the absence of a line blanketing opacity and the relative transparency of the supernova in continuum opacity that causes the lack of flux in the $1.2 \mu \mathrm{m}$ region. The work we report here supports the interpretation that a lack of line blanketing contributes to the paucity of flux at $\mathrm{J}$ with respect to $\mathrm{H}$ and puts that interpretation in a broader context.

With the interpretation that some portions of the IR have relatively large line blanketing and others relatively little, the infrared region of the spectrum provides a unique window into the supernova. In adjacent wavelength regions the supernova ejecta can be probed both deeply within the ejecta where a paucity of lines provides a minimum in the opacity and at shallow depths where strong line blanketing provides a quasi-continuum formed at relatively large radii. In this paper, we explore the formation and evolution of the spectra of SN Ia throughout the wavelength range from 0.9 to $2.5 \mu \mathrm{m}$ from about a week before to about two weeks after maximum light. We also elucidate the relevant physical diagnostics at different wavelengths and phases. In $\S 2$ we describe some observations available to us which provide a basis for comparison of the models. In $\S 3$ we describe the models used to probe the physics. In $\S 4$ we confront the theoretical results with observations. It is important to note that we are not attempting to provide a best fit to the limited available data. The objects for which we have some data are not necessarily garden variety SN Ia. In $\S 5$, we provide a final discussion and conclusions.

\section{Observations}

A limited number of existing early time IR spectra exist. The data reproduced here have been taken from the literature (Meikle et al. 1996; Bowers et al. 1997).

The data for SN 1986G were provided by Graham (private communication). SN 1986G exploded in the dust lane of Centaurus A and exhibited bluer infrared colors and shorter timescales for spectral variations than a "normal" SN Ia (Frogel et al. 1987). The spectrum shown in Figure 1 was obtained at the UKIRT 3.8-m telescope on three consecutive nights in 1986, May 23, 24 and 25. The B maximum light occurred on 1986, May $11 \pm 1$ and the V maximum light was attained 2-3 days later (Phillips et al. 1987).

SN 1994D is the most normal SN Ia for which good IR data exist (Meikle et al. 1996). Although premaximum light data are available in all the nearinfrared bands, only the $J$-band was followed significantly past the first epoch of observation. The first spectrum was obtained about 8.5 days before B maximum.

\section{Description of the Numerical Methods}

\subsection{Hydrodynamics}

The explosions are calculated using a one-dimensional radiation-hydro code, including nuclear networks (Höflich \& Khokhlov 1996, and references therein). This code solves the hydrodynamical equations explicitly by the piecewise parabolic method (Collela \& Woodward 1984) and includes the solution of the frequency- 


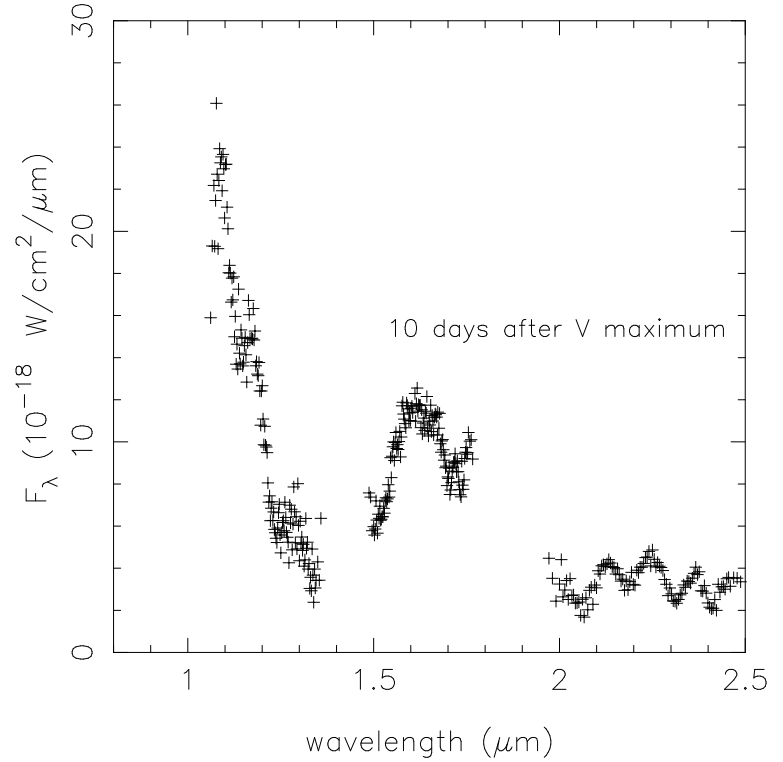

Fig. 1.- Spectrum of SN 1986G $10 \mathrm{~d}$ after visual maximum

averaged radiation transport implicitly via moment equations, expansion opacities, and a detailed equation of state. The frequency-averaged variable Eddington factors and mean opacities are calculated by solving the frequency-dependent transport equations. About one thousand frequencies (in one hundred frequency groups) and about five hundred depth points are used. Nuclear burning is taken into account using a network which has been tested in many explosive environments (see Thielemann, Nomoto \& Hashimoto 1996, and references therein).

\subsection{Spectral Calculations}

Both the LTE (Wheeler \& Harkness 1990) and nonLTE (Höflich, Wheeler \& Thielemann 1997 and references therein) codes solve the relativistic radiation transport equations in a comoving frame. Neither code fixes the luminosity of the supernova using the so-called "light bulb" approach. Rather, the energetics of the model are calculated. Given an explosion model, the evolution of the spectrum is not subject to any tuning or free parameters.

The non-LTE spectra are computed for various epochs using the chemical, density and luminosity structure and $\gamma$-ray deposition resulting from the light curve code (Höflich, Wheeler, \& Thielemann 1997, and references therein), thus providing a tight coupling between the explosion model and the radiative transfer. The effects of instantaneous energy deposition by $\gamma$-rays, the stored energy (in the thermal bath and in ionization) and the energy loss due to the adiabatic expansion are taken into account. The radiation transport equations are solved consistently with the statistical equations and ionization due to $\gamma$ radiation for the most important elements $(\mathrm{C}, \mathrm{O}, \mathrm{Ne}$, $\mathrm{Na}, \mathrm{Mg}, \mathrm{Si}, \mathrm{S}, \mathrm{Ca}, \mathrm{Fe}, \mathrm{Co}, \mathrm{Ni})$. About $10^{6}$ additional lines are included assuming LTE-level populations. The scattering, photon redistribution, and thermalization terms are computed with an equivalent-twolevel formalism that is calibrated using NLTE models (Höflich, 1995). Typically, the photon redistribution and thermalization terms are $\sim 10^{-2}$ to $10^{-3}$ in the optical and $\sim 0.1$ in the IR.

The LTE spectra are computed using similar input models and also using a consistent energy deposition calculation. The effects of instantaneous energy deposition, stored energy, and the energy loss due to the expansion are all taken into account using the approximation of flux limited diffusion. The main differences between the two codes are the energy level populations and the treatment of line scattering, photon redistribution and thermalization. For the LTE calculations, the thermalization parameter is assumed to be 0.01 , independent of frequency.

\subsection{General Results}

Explosion models have been taken from the literature. No specific tuning of the input explosion models has been made to produce a best fit to the data and the radiation transport codes were run blind. No knowledge of the data was available at the time of the code execution. Slightly different explosion models have been used in the two spectral synthesis calculations. For the LTE code model DD21 (Höflich, Wheeler \& Thielemann 1997) was used while for the non-LTE calculation model DD200 (Höflich, Khokhlov \& Wheeler 1997) was used. Both are delayed detonation models. Investigation of other explosion models lies outside the scope of this work. In DD21 the central density of the $\mathrm{C} / \mathrm{O}$ white dwarf and the density of transition from deflagration to detonation were $\approx 20$ percent higher than in DD200, making DD21 a bit more luminous due to a higher ${ }^{56} \mathrm{Ni}$ production. Figures 2 and 3 show the composition structure in velocity space for DD200 and DD21. The velocities of the intermediate mass elements, especially silicon, are somewhat different in the two models.

Figure 4 gives the evolution of the spectrum for 


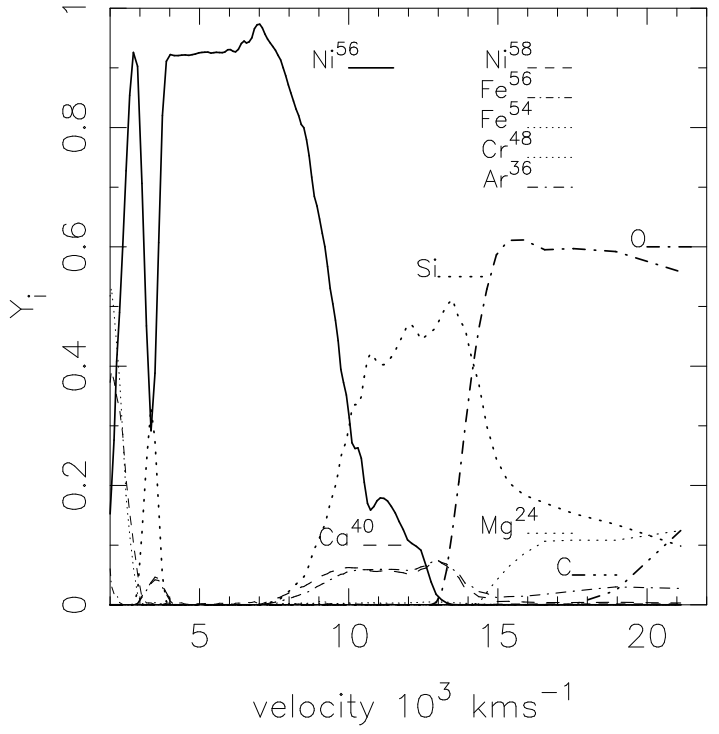

Fig. 2.- The composition of DD200

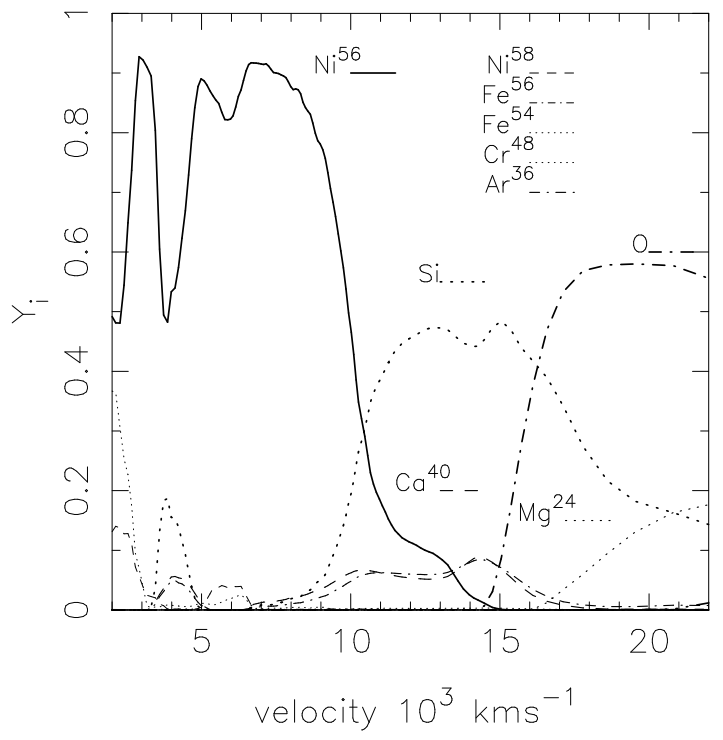

Fig. 3.- The composition of DD21
DD200. The epochs are given with respect to model V maximum. They are $-3,+7$ and +14 days corresponding to about 15,25 , and 32 days after the explosion. The spectra have been normalized to the maximum flux in the displayed range and then a constant has been added for clarity of display. The levels of zero flux for the spectra after maximum light are shown as straight lines in the red part of the spectrum.

Figure 5 presents the evolution of the radius at which of the optical depth is unity for the non-LTE model. The wavelength attributed to each radius is that of the principle ray along the line of sight to the observer and therefore the wavelengths of the radius peaks are by default blue shifted with respect to the corresponding peaks in the spectrum. The plots for +7 and $+14 \mathrm{~d}$ have been shifted upward for clarity. The levels of zero radius for those epochs are shown by the straight lines on the right side of the diagram.

Three days before $\mathrm{V}$ maximum, the spectrum in Figure 4 is relatively featureless with the exception of MgII, CaII and Si II lines at 1.05, 1.12 and 1.65 $\mu \mathrm{m}$, respectively. The opacities are dominated by electron scattering. Later on, at wavelengths shorter than $\sim 1.2 \mu \mathrm{m}$, the spectra are dominated by blends of $\mathrm{Mg}, \mathrm{Ca}$ and iron group elements. The change of the slope of this region with time provides a good test for the epoch of the observed supernova spectrum. With increasing time, the atmosphere becomes cooler. Once the electron scattering opacity decreases substantially, a SN Ia no longer has a well defined photosphere (Wheeler \& Harkness 1990, Jeffery et al. 1992, Höflich et al. 1993, Spyromilio, Pinto \& Eastman 1994). The depth of the photosphere varies with wavelength depending on the opacity of transitions present in that frequency interval (Fig. 5). The features at about $1.5-1.7$ and $2.2-2.6 \mu \mathrm{m}$ are primarily due to iron group elements of the second ionization stage with a dominant contribution from Co and a substantial contribution from Ni. The features at $2.2-2.6 \mu \mathrm{m}$ also contain appreciable contributions from intermediate mass elements, especially $\mathrm{Si}$. The small bump in the spectrum corresponding to 3 days before maximum at $1.7 \mu \mathrm{m}$ in Figure 4 is from Si II. These features appear in "emission" because they are formed at a larger radius with corresponding larger effective area. In addition, the source function is not scattering dominated, but governed by the redistribution of energy from higher frequencies (Höflich 1995). In contrast to the optical, in the IR photons are trapped within the broad opacity bands that keep 
the mean photon density and, thus the source function, higher than simple geometrical dilution of the radiation field would give.

Flux minima occur where the spectrum is formed at a small radius (Fig. 5). Again, a principle effect is that the emitting area is small. At -3 days the total continuum optical depth is about 30 . By 14 days after maximum light the continuum has decreased substantially. At this time, electron scattering, free-free and bound-free opacity give an optical depth in the continuum of $\sim 3$. At $1.2 \mu \mathrm{m}$ line blanketing is only about 20 percent of the total opacity, whereas line blanketing dominates the opacity at 1.6 and $1.8 \mu$.

The peaks and valleys in the opacity distribution may be used as a tool for analyzing the composition of the ejecta. The broad holes between 1.2 and 1.5 and 1.9 and $2.1 \mu \mathrm{m}$ correspond to a dispersion in velocity of 70,000 and $30,000 \mathrm{~km} \mathrm{~s}^{-1}$, far exceeding the dispersion of the expansion velocity of radioactive material in any supernovae. Consequently, these gaps cannot be eliminated by velocity smearing and should in general be visible when the continuum opacity is low enough to expose them. The existence of the gaps provides the opportunity to probe very different layers of the ejecta at a given time. This makes the IR an important complement to the optical and the UV.

As mentioned above, the peaks at about 1.6 and $1.8 \mu \mathrm{m}$ are due to iron peak elements, specifically lines of $\mathrm{Ni}$ and Co. They thus sample the conditions of the radioactive elements. The narrow minimum seen at $1.7 \mu \mathrm{m}$ is due to a lack of strong blended lines in this wavelength range. Both the blue and red edges of this feature are formed in layers expanding towards the observer and, consequently, blueshifted according to the maximum velocity of the radioactive material. This flux minimum can be smeared out by velocity shifts of about $10,000 \mathrm{~km} \mathrm{~s}^{-1}$. Such shifts are comparable with the expansion velocity of the radioactive material, therefore this narrow gap is a valuable diagnostic tool for the velocity spread of the region containing the radioactive material in which the feature is formed. Because the envelope becomes more transparent with increasing time exposing a broader range of velocities, the velocity spread of the visible iron group elements increases. This causes the $1.7 \mu \mathrm{m}$ gap to becomes narrower with time (Fig. 4). Clearly, the time evolution and the strength of the $1.7 \mu \mathrm{m}$ gap will depend sensitively on the underlying model.

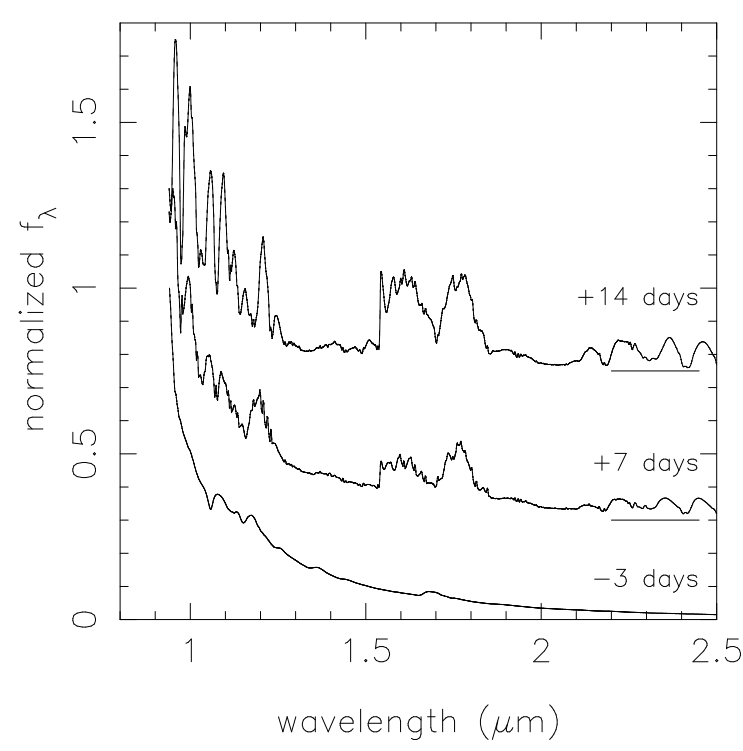

Fig. 4.- Evolution of the DD200 model spectrum (times with respect to model $\mathrm{V}$ maximum)

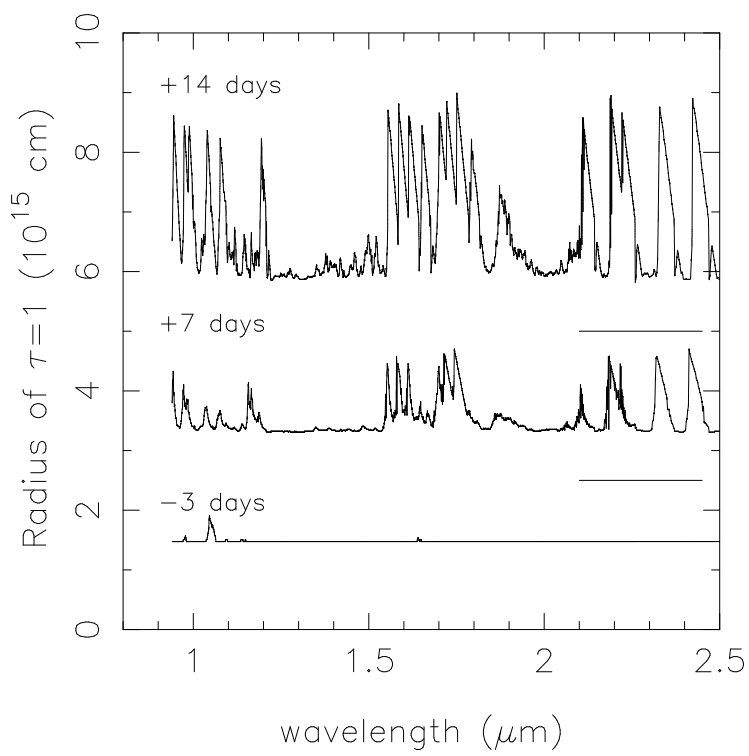

Fig. 5.- Evolution of the DD200 radius of optical depth unity 


\section{Comparison with the Observations}

We neither tuned the models to provide a best fit nor reproduced the exact times of the observations. Instead, the goal of this section is to demonstrate that the IR is a valuable tool to analyze SN Ia and to test whether our models can provide a reasonable explanation for the observations.

\subsection{Pre-maximum Spectra}

The premaximum data on SN 1994D are compared with the pre-maximum non-LTE models for DD200 in Figure 6. The data were obtained $8.5 \mathrm{~d}$ before $\mathrm{B}$ maximum light while the theoretical spectrum is 2 and $3 \mathrm{~d}$ before $\mathrm{B}$ and $\mathrm{V}$ maximum light, respectively. In this model, the strong feature in the model at 1.05 $\mu \mathrm{m}$ is due to $\mathrm{Mg}$ II and not to the helium triplet transition. The He abundances in DD200 and DD21c are rather typical for delayed detonations and the deflagration model W7. Neither has sufficient helium in the ejecta to form the line. Moreover, magnesium is a natural product of the explosive nucleosynthesis and therefore a more plausible candidate. As can be seen in the composition of DD200 (see Figure 2), magnesium is present almost uniformly (in fractional mass) in the outer layers. Early in the spectral evolution as the photosphere recedes through the outer layers, the Mg II transition is expected to be observed with decreasing velocity as measured by the absorption minimum. The Doppler shift of Mg II will cease changing as soon as the photosphere has receded below the inner edge in the distribution (Fig. 2). After that phase, the Doppler shift will not follow the receding photosphere, but remain at an almost constant velocity. Thus, early spectra provide a sensitive tool to determine the boundary between explosive carbon burning and explosive oxygen burning in velocity space. In DD200, the velocity of the onset of this transition is about $15,000-16,000 \mathrm{~km} \mathrm{~s}^{-1}$ and the photosphere has receded inside this region $\approx$ one week before maximum. Therefore, the subsequent small change in the line shift in the models is consistent with the observations (Meikle et al. 1996). Although the model agrees reasonably well with the data, the blueshift in the model is somewhat smaller than in the observations, indicating that the onset of explosive oxygen burning took place at a slightly smaller velocity in the model than in the supernova.

In Figure 7 the LTE model of DD21 is compared with the data at approximately one week before max- imum light. The model produces the MgII feature although this model exhibits a higher blueshift than the data. As can be seen in Figure 3 the magnesium in DD21 is truncated at higher velocities than in DD200.

In the DD200 model spectrum a relatively strong line can be seen at $1.15 \mu \mathrm{m}$ (Fig. 6). This is due to CaII. In Figure 2 calcium is found at the interface layer between the burning to nickel and the burning to intermediate mass elements. The absence of the feature in the data can be attributed to the fact that the model represents a phase $\sim 6$ days later than the data when the scattering photosphere has moved further inwards in the model. The appearance of this feature is thus a useful diagnostic of the location of the transition layer between complete and incomplete burning of silicon. In SN1994D, a feature at approximately the right wavelength but without the clear P-Cygni signature becomes visible about $1-2 \mathrm{~d}$ past maximum (Meikle et al. 1996). We note that the optical Ca II features cannot provide this diagnostic. In that wavelength regime, primordial (not freshly synthesized) calcium contaminates the ejecta and provides sufficient lines of significant optical depth to confuse the interpretation. The DD21 LTE model spectrum does not show a strong Ca II line at 1.15 $\mu \mathrm{m}$. The difference between the two models can be understood as being due to the difference in epoch and the difference in the structure of the exploding star.

The P-Cygni feature at $1.67 \mu \mathrm{m}$ observed in the SN 1994D data is reasonably reproduced in both models (Fig. 7). In the models, this feature is due to Si II. Since silicon is produced in all layers above $8,500 \mathrm{kms}^{-1}$, the Doppler shift of Si II follows the photosphere. Clearly the photospheric velocity from the models is not perfectly matched to the data. This feature is less likely to be present in a supernova such as SN 1991T. In overluminous supernova such as SN 1991T less silicon is made and it is hotter and therefore probably in a higher ionization state.

Both the model and the data are fairly unexciting in the $K$-band $(2.2 \mu \mathrm{m})$ prior to maximum light. A small feature is present in the data at $2.05 \mu \mathrm{m}$. A similar, but somewhat weaker, spectral feature is also present in the LTE model and is due to SiIII. This feature also appears as an opacity source in the non-LTE model, but does not cause a strong feature because, at the later epoch of the model, the main ionization stage is Si II. 

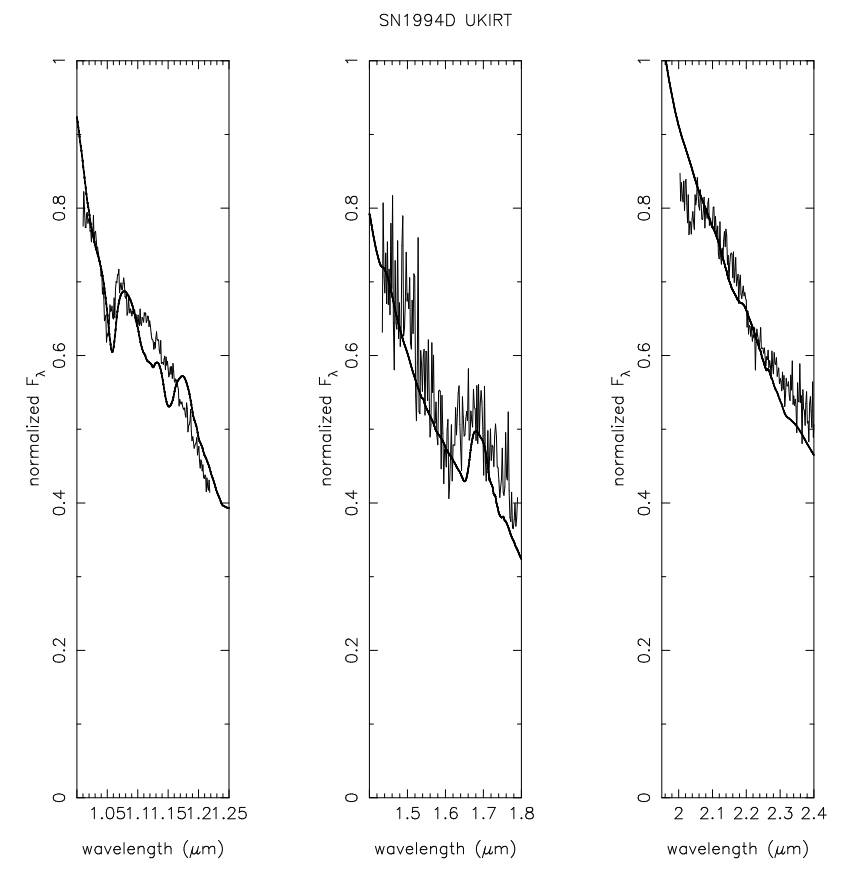

\subsection{Post-maximum Spectra}

In Figures 8-10 we compare the data from SN 1986G with the models. Note that SN $1986 \mathrm{G}$ is regarded to be a somewhat subluminous, rapidly declining event compared to "normal" SN Ia. We have not attempted to select models specifically appropriate for these circumstances, but can address general features and possible diagnostics of such supernovae.

Overall, the non-LTE models produce the observed spectral features rather well. The non-LTE theoretical spectra in Figures 8 and 9 corresponding to about +7 and $14 \mathrm{~d}$ after model $\mathrm{V}$ maximum light (a day later than model B maximum) bracket the epoch of observations at $10 \mathrm{~d}$. Within the uncertainties, the differences between the data and the model spectra can be attributed to the time evolution. The LTE model 24 days after the explosion corresponds to about one week after model V maximum light in DD21 (Fig. 10). The ratio of the dips in the spectra at 1.2 and $1.9 \mu \mathrm{m}$ and the quasi-continuum in the $H$-band evolve dramatically in the models. This can be expected as the continuum opacity drops. The iron-group elements are exposed and the associated strong line blanketing causes the photosphere to form at large radii (Fig. 5) while the lack of opacity in the holes leaves the core exposed. The LTE model in Figure 10 does not reproduce the J-band spectral minimum at $\sim 1.2 \mu \mathrm{m}$. This model gives a "continuum" at this wavelength and epoch that is caused by line blanketing due to $\mathrm{Si}$ I. In the non-LTE model, most of the silicon is in the second ionization stage and there is very little Si I. The difference can be attributed to the fact that, in the LTE models, the matter temperature is given by the energy density and drops strongly with radius as $T \propto R^{-1 / 2}$. In the non-LTE models, the matter temperature more closely follows the radiation temperature (see also Jeffery et al. 1992). This implies that the $1.2 \mu \mathrm{m}$ hole provides a temperature diagnostic for the silicon layers.

Frogel et al. (1987) suggested that the minimum observed in their IR spectra of SN 1986G at $1.7 \mu \mathrm{m}$ could be identified as magnesium with a redshift of -0.0025 . Note that a negative redshift implies that Frogel et al. used the depressions in the data to identify the lines. The narrow dip at $1.7 \mu \mathrm{m}$ in both models presented here is due to a lack of line opacity. This obvious feature in the data for SN 1986G is not visible in the data from SN 1991T (see Bowers et al. 1997). As discussed in $§ 3.2$, a plausible explana- 


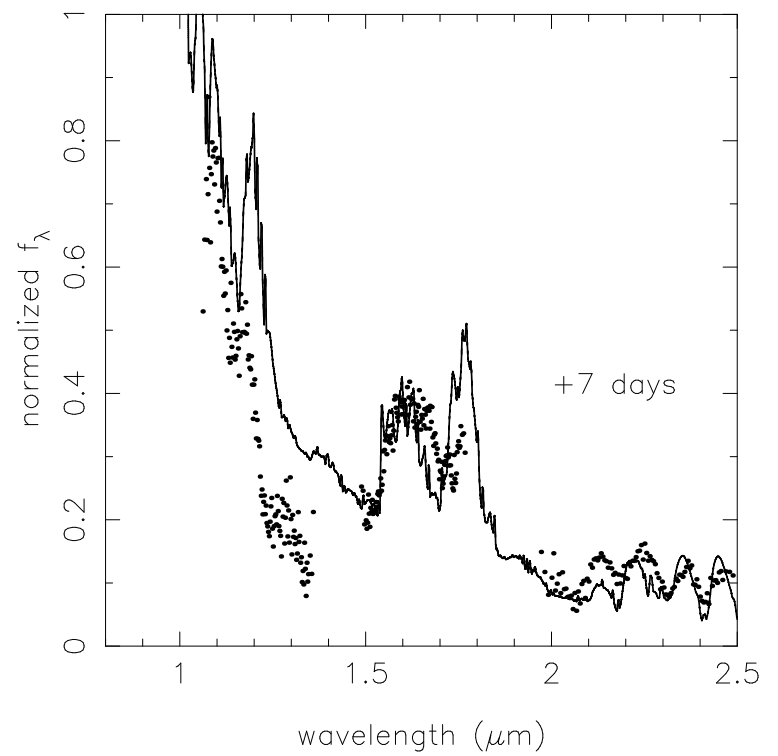

Fig. 8. - non-LTE model at $+7 \mathrm{~d}$ and SN $1986 \mathrm{G}$ at $+10 \mathrm{~d}$ with respect to model and observed $\mathrm{V}$ maximum, respectively.

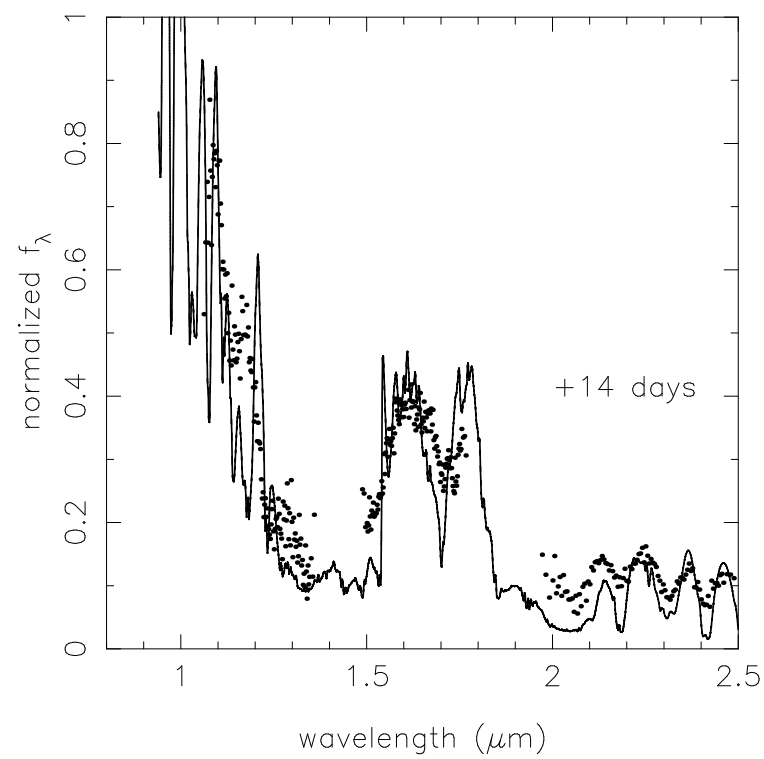

Fig. 9. - non-LTE model at $+14 \mathrm{~d}$ and SN $1986 \mathrm{G}$ at $+10 \mathrm{~d}$ with respect to model and observed $\mathrm{V}$ maximum, respectively.

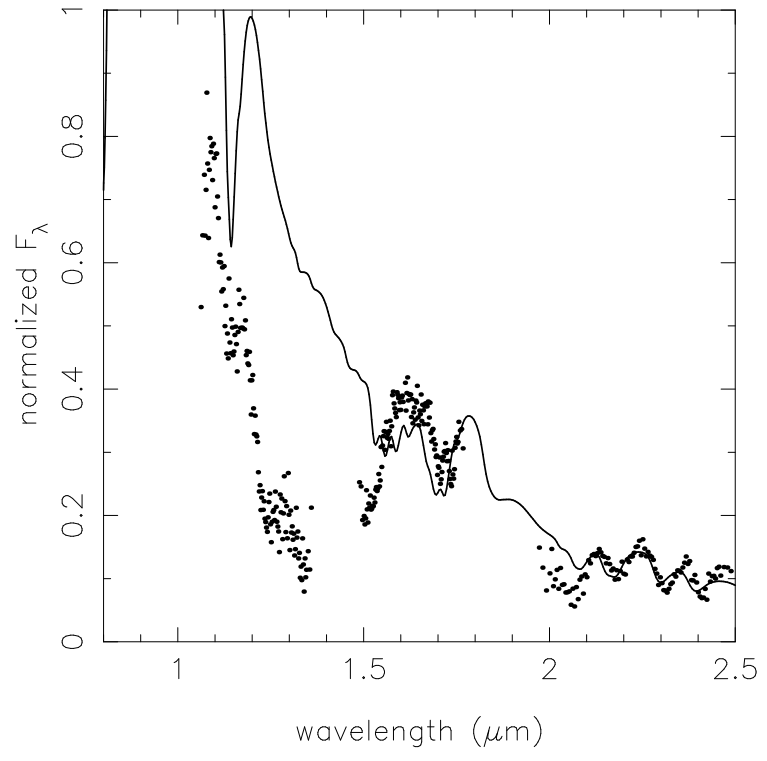

Fig. 10. - LTE model at 24 days after explosion ( $\approx+7 \mathrm{~d}$ after model $\mathrm{V}$ maximum) and SN 1986G at $+10 \mathrm{~d}$ past $\mathrm{V}$ maximum.

tion for the difference is that the luminous SN 1991T has $\mathrm{Ni} / \mathrm{Co}$ at higher velocities, thus smearing out the opacity gap at $1.7 \mu \mathrm{m}$.

As mentioned above, the strong quasi-continuum in the $H$-band $(1.6 \mu \mathrm{m})$ is due to a large number of iron group lines. Although iron underlies most of the emission, the bluer of the two broad features in the models is dominated by cobalt transitions while nickel contributes strongly to the redder of the two. In part, the evolution of the ratio of the two features can be attributed to the increase of the cobalt abundance relative to the nickel due to the radioactive decay of the nickel to cobalt. The smaller red shift of the flux minimum at about $1.7 \mu \mathrm{m}$ in both models DD200 and DD21 in comparison with the observations $\left(\sim 1,500 \mathrm{~km} \mathrm{~s}^{-1}\right)$ could be accounted for by the fact that SN 1986G was slightly subluminous (see Phillips et al. 1987) and had less nickel expanding at a smaller velocity than the "normal" models compared here.

Frogel et al. (1987) identified the $K$-band $(2 \mu \mathrm{m})$ features as due to NaI with a redshift of -0.00133 . The spectral models presented here identify these features as being due to transitions of iron-peak ele- 
ments and silicon. The $K$-band features are present in both the SN 1986G and SN 1991T data (Bowers et al. 1997). They are well reproduced by both the LTE and non-LTE models. These features can be expected to be present in all SN Ia spectra although their velocities may vary. In the models, these features are formed in the transition region between complete and incomplete silicon burning. The overlap between these regions is sensitive to the explosion model and the $K$-band features can be used as a diagnostic of the composition structure of the two regions that can be clearly seen in Figures 2 and 3 .

Overall, both the LTE and non-LTE models reproduce the strong features because the basic pattern depends on the atomic physics and the structure of the explosion model, rather than the level populations.

\section{Discussion and Conclusions}

The near-IR wavelength range has been shown to be a valuable tool to diagnose explosion models of SN Ia. The current generation of atmosphere codes is well suited to allow quantitative analysis of the observed spectra by means of delayed detonation models. The delayed detonation scenario works well both for the optical and IR. The good reproduction of the IR is an especially satisfactory result given that the calculations were done without prior knowledge of the observations and without "tuning" the dynamic models or the atomic data, but taking the results as they were. These models provide a good basic understanding of the IR spectra. The IR is thus revelaed as a unique tool providing spectral diagnostics of basic quantities such as the boundary between explosive carbon and oxygen and between complete and incomplete silicon burning by measuring $\mathrm{Mg}$ II, Ca II and iron group lines.

This paper concentrated on the IR spectral features, but a word about the IR photometric evolution is also relevant. Elias et al. presented H-band light curves and the evolution of the J-H color with time and showed that SN Ia follow a typical photometric evolution. $\mathrm{J}$ and $\mathrm{H}$ peak about 6 days before $\mathrm{B}$ maximum (note that all the figures in Elias et al. are given in terms of time since $\mathrm{H}$ maximum, not $\mathrm{B}$ maximum). The $\mathrm{H}$ light curve has a pronounced minimum about 9 days and $\mathrm{J}$ about 14 days after B maximum. Both $\mathrm{J}$ and $\mathrm{H}$ have a secondary flux maximum about 24 days after $\mathrm{B}$ maximum. The J-H color curve reddens dramatically from about 0 near optical maximum to a maximum of about 1.414 days after B maximum. It then turns blueward to a value of about 0.3 a little over 30 days past B maximum and finally moves redward again to a constant value of about 1.7 after 80 days. Similar non-monotonic light curve features with secondary bumps or inflections appear in other photometric bands from the $\mathrm{V}$ to the $\mathrm{K}$ band with varying intensities (Suntzeff et al. 1998). Elias et al. argued that the $J$-band light curve minimum resulted from an absorption rather than a later increase in emission at the time of the second IR maximum that corresponds approximately to the onset of the exponential decay in the optical light curve at 30 days past maximum. Graham (1986) attributed the red J-H color of SN Ia to the strong "absorption" at 1.2 $\mu \mathrm{m}$. The current models suggest that Graham was basically correct about the overall reddening of J-H. The lack of flux at $1.2 \mu \mathrm{m}$ compared to the increased emission due to the strong quasi-continuum in $\mathrm{H}$ certainly makes the J-H color red. Graham bolstered his argument, however, by drawing a parallel between the behavior of the $\mathrm{J}-\mathrm{H}$ color curve and the strength of absorption of the $\mathrm{Na} \mathrm{D}$ line. The latter may not be reliable because in the relevant time frame, the [Co III] line is suspected to begin to yield net emission at just the wavelength of the $\mathrm{Na}$ line. In any case lacking a model, Graham did not actually attempt to account for the non-monotonic behavior of the $\mathrm{J}$ and $\mathrm{H}$ band light curves with their distinct secondary maxima.

Höflich, Khokhlov, \& Wheeler (1995) have presented an explanation for the broad band non-monotonic behavior of the IR light curves in which time dependent opacity can lead to an effective emitting radius and hence area that peaks at a delayed time in the IR compared to the optical. Thus their explanation is just the opposite of that of Elias et al. (1985). The non-monotonic IR light curves are due to a later increase in flux, not an intermediate "absorption. In these models, the $1.2 \mu \mathrm{m}$ spectral "hole" does not play a direct role in the light curve evolution. The $1.2 \mu \mathrm{m}$ spectral feature is undoubtedly the basic source of the very red colors of SN Ia after maximum, but its presence does not yield a direct explanation for the nonmonotonic behavior of either the J band light curve nor the evolution of the $\mathrm{J}-\mathrm{H}$ color. This issue is surely not yet closed. We note that despite their success, the models of Höflich et al. predict the first peak in $\mathrm{J}$ and $\mathrm{H}$ to occur quite close to $\mathrm{B}$ maximum, rather than 6 days earlier as the data seem to suggest.

Finally, we want to stress that this paper should 
not be regarded as a final answer, but a first step toward the analysis of the IR spectra of Type Ia supernovae. To utilize the full potential of the IR, detailed analysis of IR spectra together with optical spectra and light curves are essential to answer questions concerning the structure of Type Ia supernova.

\section{ACKNOWLEDGMENTS}

James Graham, Peter Meikle and Emma Bowers are thanked for providing data in digital form and Eddie Baron, David Branch, Peter Meikle, Ken Nomoto, and Douglas Swartz for comments on the manuscript. JS gratefully acknowledges the hospitality of the University of Texas at Austin, where this work was started. JCW, PAH and JS would like to thank the Institute of Theoretical Physics at the University of California at Santa Barbara, where this work was finished. The ITP is supported by the NSF under Grant No. PHY94-07194. This research was supported in part by NSF Grant AST 9528110, NASA Grant NAG 5-2888, and a grant from the Texas Advanced Research Program,

\section{REFERENCES}

Bowers, E.J.C. et al., 1997, MNRAS, (in press)

Branch, D. et al. 1983, ApJ, 270, 123

Collela P., Woodward P.R., 1984, J.Comp.Phys., 54, 174

Elias J.H., Matthews M., Neugebauer G., Person S.E., 1985, ApJ, 296, 379

Frogel J.A. et al., 1987, ApJ, 315, L129

Graham J.R., 1986, MNRAS, 220, 27p

Höflich P., 1995, ApJ, 443, 89

Höflich P., Khokhlov A., 1996, ApJ, 457, 500

Höflich P., Khokhlov A., Wheeler, 1995, ApJ, 444, 831

Höflich P., Khokhlov A., Wheeler, 1997, ApJ, in press

Höflich P., Müller E., Khokhlov A., 1993, A\&A, 268, 570

Höflich P., Wheeler J.C., Thielemann F., 1997, ApJ, (submitted)
Jeffery D.J., Leibundgut B., Kirshner R.P., Benetti S., Branch D., Sonneborn G., 1992, ApJ, 397, 304

Kirshner, R. P., Willner, S. R., Becklin, E. E., Neugebauer, G., \& Oke, J. B. 1973, ApJ, 180, L97

Lynch D.K., Rudy R.J., Rossano G.S., Erwin P., Puetter R.C., Branch D., 1990, AJ, 100, 223

Meikle W.P.S. et al., 1996, MNRAS, 281, 263

Phillips M.M. et al., 1987, PASP, 99, 592

Spyromilio J., Pinto P.A., Eastman R.G., 1994, MNRAS, 266, L17

Suntzeff, N. et al. 1998, in preparation.

Thielemann F.K., Nomoto K., Hashimoto M., 1996, ApJ, 460, 408

Wheeler J.C., Harkness R.P., 1990, Rept. on Prog. in Phys., 53, 1467

This 2-column preprint was prepared with the AAS LATEX macros $\mathrm{v} 4.0$. 